\title{
Spin splitting and precession in quantum dots with spin-orbit coupling: The role of spatial deformation
}

\author{
Manuel Valín-Rodríguez, ${ }^{1}$ Antonio Puente, ${ }^{1}$ and Llorenç Serra ${ }^{1,2}$ \\ ${ }^{1}$ Departament de Física, Universitat de les Illes Balears, E-07122 Palma de Mallorca, Spain \\ ${ }^{2}$ Institut Mediterrani d'Estudis Avançats IMEDEA (CSIC-UIB), E-07122 Palma de Mallorca, Spain \\ (Received 28 May 2003; revised manuscript received 28 August 2003; published 13 February 2004)
}

\begin{abstract}
Extending a previous work on spin precession in GaAs/AlGaAs quantum dots with spin-orbit coupling, we study the role of deformation in the external confinement. Small elliptical deformations are enough to alter the precessional characteristics at low magnetic fields. We obtain approximate expressions for the modified $g$ factor including weak Rashba and Dresselhaus spin-orbit terms. For more intense couplings numerical calculations are performed. We also study the influence of the magnetic-field orientation on the spin splitting and the related anisotropy of the $g$ factor. Spin-orbit coupling effects can reproduce the experimental spin splittings reported by Hanson et al. [Phys. Rev. Lett. 91, 196802 (2003)] for a one-electron dot. For dots containing more electrons, Coulomb interaction effects are estimated within the local-spin-density approximation, showing that many features of the noninteracting system are qualitatively preserved.
\end{abstract}

DOI: 10.1103/PhysRevB.69.085306

PACS number(s): 73.21.La

\section{INTRODUCTION}

In the last decade the study of spin-related phenomena has become one of the most active research branches in semiconductor physics. The present advances in spin-based electronics ${ }^{1}$ and the hope for better devices, with enhanced performance with respect to the conventional charge-based ones, encourage this research. Two physical mechanisms underlie the operation of most spintronic devices: (a) the spinspin interaction, present in ferromagnetic materials and in diluted magnetic semiconductors; and (b) the electron spinorbit (SO) coupling stemming from relativistic corrections to the semiconductor Hamiltonian. It should also be mentioned that, as shown recently by Ciorga et al., another possibility of spin control involves the use of external magnetic fields to induce changes in the spin structure of a quantum $\operatorname{dot}^{2}$ These spin modifications affect the passage of currents through the system, originating the spin blockade effect. A conspicuous example of a device exploiting the SO coupling is the spin transistor, first proposed by Datta and Das. ${ }^{3}$ In this system the spin rotation induced by an adjustable Rashba coupling is used to manipulate the current.

In a recent work, ${ }^{4}$ we studied the spin precession of quantum dots with SO coupling under the action of a vertical magnetic field of modulus $B$. It was shown that the SO coupling modifies the precessional frequency from the Larmor expression $\hbar \omega_{L}=\left|g^{*}\right| \mu_{B} B$, where $g *$ is the bulk effective $g$ factor and $\mu_{B}$ is the Bohr magneton, to a different value depending on the dot quantum state. Namely, the modified precessional energy equals the gap between spin-up and spin-down states for the active level, the so-called spin-flip gap $\Delta_{s f}$.

Purely circular dots are characterized by discontinuous jumps in angular momentum with the number of electrons $N$ and the magnetic field, with a similar behavior for the precessional frequency. An interesting prediction of Ref. 4 was that for some values of $N$ a finite $\Delta_{s f}$ persists even at $B$ $=0$, i.e., a constant offset to the above Larmor formula. It is our aim in this work to extend those investigations by including deformation in the external confinement, as well as a more general treatment of the SO coupling, considering Rashba and Dresselhaus contributions on an equal footing. We shall show that small elliptical deformations are enough to sizably alter the precessional frequency, yielding a deformation-dependent $g$ factor and washing out the low $B$ offsets of purely circular dots. Anisotropy effects in the $g$ factor will also be studied by allowing for a tilted orientation of the magnetic-field vector with respect to the dot plane.

Spin dynamics in semiconductor nanostructures can be experimentally monitored with optical techniques. Indeed, a time-delayed laser interacting with a precessing spin experiences the Faraday rotation of its polarization. Measuring the rotation angle for different delays allows to map the spin orientation and thus observe in detail the dynamics. This technique has been applied to bulk semiconductors (see Ref. 5 for a recent review) and, also, to CdSe excitonic quantum dots in Ref. 6. Alternative methods to gather information on the $g$ factor in quantum dots normally use measurements of the resonant tunneling currents through the system that permit the determination of the spin splittings and, therefore, deduce the effective $g$ value. ${ }^{7,8}$

Electron spin in quantum dots is much more stable than in bulk semiconductors, due to the suppression of spin-flip decoherence mechanisms. ${ }^{9}$ Spin relaxation is predicted to occur on a time scale of $1 \mathrm{~ms}$ for $B=1 \mathrm{~T}$. Accordingly, in this work we shall neglect spin relaxation, focussing on the much faster spin precession in quantum dots. The spin splittings will be compared with those measured in Ref. 8, showing that SO coupling effects can indeed reproduce the observed behavior. The paper is organized as follows. Section II presents the analytical model for low SO intensities. In Sec. III we discuss the numerical results for a variety of situations; namely, arbitrary SO strengths (A), tilted magnetic fields (B), one-electron dots (C), and treating Coulomb interaction effects (D) within the local-spin-density approximation (LSDA). Finally, the conclusions are presented in Sec. IV. 


\section{THE MODEL}

\section{A. The noninteracting Hamiltonian}

Our model of a single quantum dot consists in $N$ electrons of effective mass $m^{*}$ whose motion is restricted to the $x y$ plane where an electrostatic potential $V_{\text {ext }}(\mathbf{r})$ induces the confinement. We assume a GaAs host semiconductor, for which $m^{*}=0.067 m_{e}$. To allow for elliptically deformed shapes we consider an anisotropic parabola, i.e.,

$$
V_{e x t}(\mathbf{r})=\frac{1}{2} m^{*}\left(\omega_{x}^{2} x^{2}+\omega_{y}^{2} y^{2}\right)
$$

Neglecting for the moment Coulomb interactions between electrons we treat the Hamiltonian for independent particles $\mathcal{H}_{i p}=\sum_{i=1}^{N} h(i)$. The single-electron Hamiltonian $(h)$ contains the kinetic/confinement energy $\left(h_{0}\right)$, the Rashba $\left(h_{R}\right)$ and Dresselhaus $\left(h_{D}\right)$ SO terms, and the Zeeman energy $\left(h_{Z}\right)$,

$$
h=h_{0}+h_{R}+h_{D}+h_{Z} .
$$

The explicit expressions of $h_{0}$ and $h_{Z}$ read

$$
\begin{gathered}
h_{0}=\frac{\mathbf{P}^{2}}{2 m^{*}}+V_{\text {ext }}(x, y), \\
h_{Z}=\frac{1}{2} g^{*} \mu_{B}\left(B_{x} \sigma_{x}+B_{y} \sigma_{y}+B_{z} \sigma_{z}\right),
\end{gathered}
$$

where $\mathbf{P}=-i \hbar \boldsymbol{\nabla}+(e / c) \mathbf{A}$ represents the kinetic momentum depending on the vector potential $\mathbf{A}=B_{z} / 2(-y, x)$ and the $\sigma$ 's are the Pauli matrices (used also in the SO contributions). Note that all three components of the magnetic field contribute to the Zeeman term while only the vertical one couples with the kinetic energy through the vector potential. The GaAs bulk $g$ factor is $g^{*}=-0.44$. Finally, the Rashba and Dresselhaus SO Hamiltonians may be written as ${ }^{10}$

$$
\begin{gathered}
h_{R}=\frac{\lambda_{R}}{\hbar}\left(P_{y} \sigma_{x}-P_{x} \sigma_{y}\right), \\
h_{D}=\frac{\lambda_{D}}{\hbar}\left(P_{x} \sigma_{x}-P_{y} \sigma_{y}\right) .
\end{gathered}
$$

The coupling constants $\lambda_{R}$ and $\lambda_{D}$ determine the SO strengths and their actual values may depend on the sample. Several experiments on quantum wells have recently provided valuable information about realistic ranges of variation for these coefficients. ${ }^{11-15}$

\section{B. The analytical solution}

It is possible to obtain analytical solutions when $h_{0}$ $\gg\left(h_{R} \simeq h_{D}\right) \gg h_{Z}$ and $B_{x}=B_{y}=0$. In this case one may use unitary transformations (as suggested in Ref. 16) yielding a diagonal transformed Hamiltonian. In a recent work ${ }^{17}$ we used this technique to show that the SO (Dresselhaus) coupling induces oscillations between up and down spin states when the magnetic field or the dot deformation are varied. Generalizing the transformations to consider both SO terms we define

$$
\begin{gathered}
\tilde{h}=U_{1}^{\dagger} h U_{1}, \\
U_{1}=\exp \left\{-i \frac{m^{*}}{\hbar^{2}}\left[\lambda_{R}\left(y \sigma_{x}-x \sigma_{y}\right)+\lambda_{D}\left(x \sigma_{x}-y \sigma_{y}\right)\right]\right\} .
\end{gathered}
$$

Expanding in powers of the $\lambda$ 's one finds for the transformed Hamiltonian

$$
\begin{aligned}
\tilde{h}= & \frac{\mathbf{P}^{2}}{2 m^{*}}+V_{e x t}(x, y)-\left(\lambda_{R}^{2}-\lambda_{D}^{2}\right) \frac{m^{*}}{\hbar^{3}} L_{z} \sigma_{z}+\frac{1}{2} g^{*} \mu_{B} B_{z} \sigma_{z} \\
& -\left(\lambda_{R}^{2}+\lambda_{D}^{2}\right) \frac{m^{*}}{\hbar^{2}}+O\left(\lambda^{3}\right)
\end{aligned}
$$

where we have defined the kinetic angular momentum operator $L_{z}=x P_{y}-y P_{x}$. Note that to $O\left(\lambda^{2}\right)$, with $\lambda$ referring to both $\lambda_{R}$ and $\lambda_{D}$, the Hamiltonian of Eq. (8) is diagonal in spin space. Nevertheless, the $x$ and $y$ spatial degrees of freedom are still coupled through the vector potential in the kinetic energy and in $L_{z}$.

A second transformation for each spin subspace of Eq. (8) may be used to obtain spatially decoupled oscillators. Namely, introducing a renormalized cyclotron frequency

$$
\omega_{c \eta}=\frac{e B_{z}}{m^{*} c}+\left(\lambda_{D}^{2}-\lambda_{R}^{2}\right) \frac{2 m^{*}}{\hbar^{3}} s_{\eta}
$$

where $s_{\eta}= \pm 1$ for $\eta=\uparrow, \downarrow$, in Eqs. (5) of Ref. 17 one obtains the masses $M_{k \eta}$ and frequencies $\Omega_{k \eta}$ of the two ( $k$ $=1,2$ ) decoupled oscillators for each spin. Analogously, Eq. (7) of that reference yields the eigenvalues $\varepsilon_{N_{1} N_{2} \eta}$, depending on the corresponding number of quanta in each oscillator $\left(N_{1}, N_{2}\right)$. For completeness of the presentation we repeat here the expressions for the latter two quantities,

$$
\Omega_{k \eta}=\frac{1}{\sqrt{2}}\left[\omega_{x}^{2}+\omega_{y}^{2}+\omega_{c \eta}^{2} \pm \sqrt{\left(\omega_{x}^{2}+\omega_{y}^{2}+\omega_{c \eta}^{2}\right)^{2}-4 \omega_{x}^{2} \omega_{y}^{2}}\right]^{1 / 2}
$$

where the upper (lower) sign in \pm corresponds to $k$ $=1(2)$, and

$$
\varepsilon_{N_{1} N_{2} \eta}=\left(N_{1}+\frac{1}{2}\right) \hbar \Omega_{1 \eta}+\left(N_{2}+\frac{1}{2}\right) \hbar \Omega_{2 \eta}+s_{\eta} \frac{1}{2} g^{*} \mu_{B} B_{z} .
$$

As a direct application of the above results we may write the effective $g$ factor for precession around a vertical magnetic field from the difference between the up and down single-particle energies $\left(\Delta_{s f}\right)$ with fixed oscillator quanta $N_{1}$ and $N_{2}$,

$$
|g| \equiv \frac{\Delta_{s f}}{\mu_{B} B}=\left|g *+\frac{\hbar}{\mu_{B} B_{z}} \sum_{k=1,2}\left(N_{k}+\frac{1}{2}\right)\left(\Omega_{k \uparrow}-\Omega_{k \downarrow}\right)\right| .
$$

This equation shows that in the general case the $g$ factor is actually a function of the electron state (through the quanta), the SO coupling constants, and the vertical magnetic field $B_{z}$ 


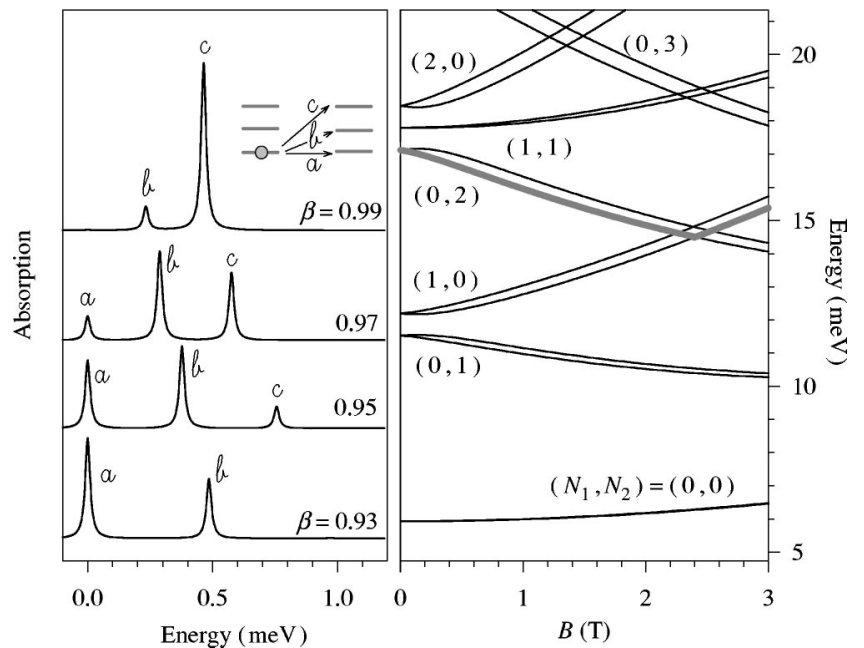

FIG. 1. (Color online) Right panel: Evolution of the singleparticle energies as a function of vertical magnetic field for a deformed quantum dot having $\beta=0.9$, where $\beta=\omega_{y} / \omega_{x}$, and a centroid value of $\hbar\left(\omega_{x}+\omega_{y}\right) / 2=6 \mathrm{meV}$. Each doublet corresponds to different spin orientations in the transformed frame (Sec. II B). The SO intensity is fixed at $\lambda_{D}^{2}-\lambda_{R}^{2}=\left(1.2 \times 10^{-9} \mathrm{eV} \mathrm{cm}\right)^{2}$. The level responsible for the spin-flip transition when $N=7$ is marked with a thick line. Left panel: Absorption strength (Ref. 18) of the spin-flip excitations at $B=0$ for different deformations ( $\beta$ 's) and the same centroid value for the parabolic confinement of the right panel. The inset characterizes the transitions for $N=7$ and $B=0$ of the right panel, with $a$ indicating the transition between Kramers conjugates.

(through the $\Omega$ 's). It is also worth mentioning that since the energy gap $\Delta_{s f}$ and the modulus of the magnetic field $(B)$ are positive quantities, only the absolute value of the $g$ factor is determined from Eq. (12).

\section{The transition between Kramers conjugates}

When B vanishes the full Hamiltonian fulfills timereversal symmetry and, according to a well-known theorem of quantum mechanics, in that limit a degeneracy should prevail (Kramers degeneracy). As shown in Fig. 1, the single-particle energies $\varepsilon_{N_{1} N_{2} \eta}$ indeed merge into degenerate pairs at vanishing magnetic field. These pairs are split by the combined action of the SO and magnetic-field contributions and for a given $\left(N_{1}, N_{2}\right)$ one obtains parallel doublets when increasing $B$. Depending on the sign of $\lambda_{D}^{2}-\lambda_{R}^{2}$ the lower member of each doublet will have a given spin orientation in the transformed frame; namely, upwards for positive sign and downwards for negative sign.

If the system has good angular momentum $\left(L_{z}\right)$ in the intrinsic reference frame, as happens in a circular confinement $\omega_{x}=\omega_{y}$, the Kramers conjugates at $B=0$ possess opposite angular momenta or, what is equivalent, reversed oscillator quanta, $\left(N_{1}, N_{2}\right)$ and $\left(N_{2}, N_{1}\right)$. Therefore, the transition between Kramers conjugates is not the spin-flip transition between levels with fixed oscillator quanta as defined above (having an energy $\Delta_{s f}$ ). In other words, the precessional transition between Kramers conjugates is forbidden in circular dots since the relevant matrix element ${ }^{18}$ preserves

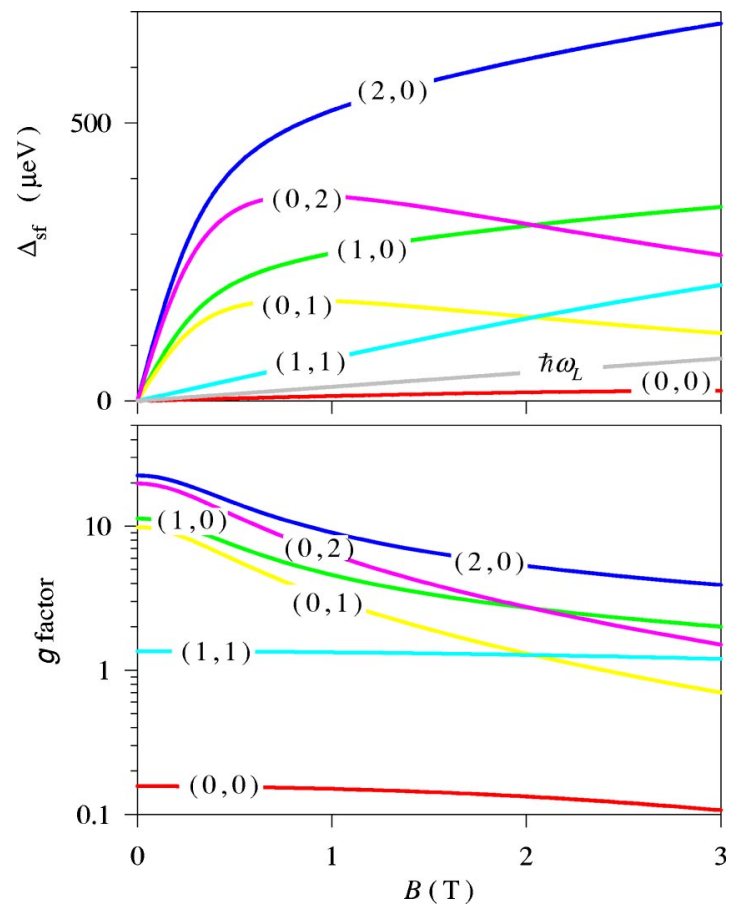

FIG. 2. (Color online) Upper panel: spin-flip energy gap for the different levels of Fig. 1 (right panel). The Larmor energy $\hbar \omega_{L}$ is also indicated. Lower panel: $g$ factors in absolute value inferred from the upper panel results using $|g|=\Delta_{s f} /\left(\mu_{B} B\right)$. Note that the vertical axis corresponds to a logarithmic scale.

angular momentum. On the contrary, when the system is deformed, for instance, due to an anisotropic confinement $\omega_{y}$ $=\beta \omega_{x}$, the Kramers-conjugates transition is the spin-flip transition, since angular momentum is no longer a "good" quantum number and, in this case, Kramers conjugates are characterized by the same oscillator quanta. This key point determines qualitatively different spin precessional spectra. In fact, when the transition between conjugates is forbidden there is a gap in the spin precession spectrum corresponding to a nonvanishing precession frequency at $B=0$ (the precessional offset discussed in Ref. 17). This gap vanishes if the transition between conjugates is allowed due to the deformation.

The left panel of Fig. 1 illustrates the discussed effect by showing the evolution of the precessional peaks as the deformation is reduced $(\beta \rightarrow 1)$. It is clearly shown that the transition between Kramers conjugates (peak $a$ ), which is a gapless (zero-energy) excitation at $B=0$, switches off when approaching the circular case. In this limit $(\beta \sim 1)$ the lower excitations (peaks $b$ and $c$ ) are at a nonvanishing energy, i.e., the system has a gap for spin-flip oscillations at vanishing $B$.

\section{The $g$ factors}

The upper panel of Fig. 2 displays the spin-flip gap for different levels, characterized by their oscillator quanta in the transformed frame. The lower panel shows the corresponding $g$ factors obtained from $\Delta_{s f}$ and the modulus of the magnetic field using the first equality of Eq. (12). As in Fig. 1 a SO value of $\lambda_{D}^{2}-\lambda_{R}^{2}=\left(1.2 \times 10^{-9} \mathrm{eV} \mathrm{cm}\right)^{2}$ as well as a defor- 


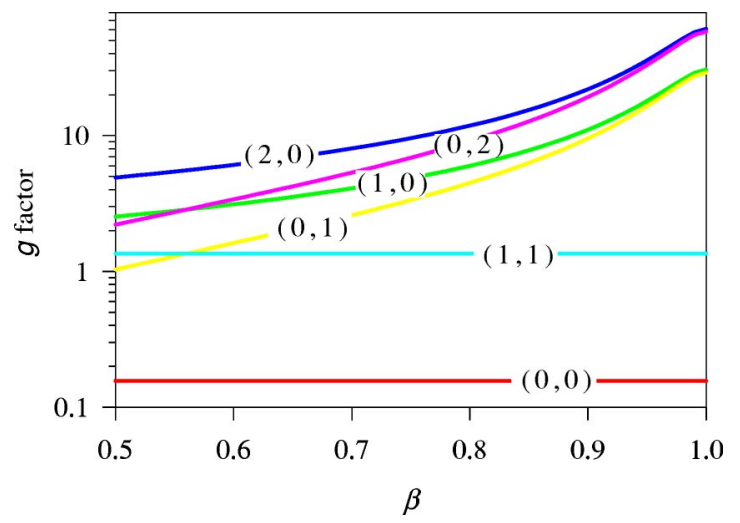

FIG. 3. (Color online) Deformation dependence of the $g$ factors for a quantum dot having $\hbar\left(\omega_{x}+\omega_{y}\right) / 2=6 \mathrm{meV}$ and $\omega_{y}=\beta \omega_{x}$ in a vertical magnetic field of $B=0.1 \mathrm{~T}$. As in Fig. 1, a SO coupling strength of $\lambda_{D}^{2}-\lambda_{R}^{2}=\left(1.2 \times 10^{-9} \mathrm{eV} \mathrm{cm}\right)^{2}$ has been assumed.

mation of $\beta=0.9$ have been assumed. We note that there is a strong dependence of the precessional properties on the electronic state, with many cases showing a dramatic deviation from the Larmor result. When the number of quanta is shared asymmetrically between the two oscillators the $g$ factor takes very large values at small magnetic fields, decreasing quite abruptly with $B$. On the contrary, when $N_{1}=N_{2}$ there is a rather flat $B$ dependence of the $g$ factor and lower enhancements. Note also that spin-flip energies below the Larmor result are obtained for the $(0,0)$ state, implying a $g$ factor lower than the bare value. We have checked that other values of the SO couplings and dot deformations do not lead to qualitative variations of this behavior, although, obviously, the numerical values are changed. The $\beta$ dependence is illustrated by the results of Fig. 3. As in Fig. 2, there is a conspicuous variation with the quantum numbers $\left(N_{1}, N_{2}\right)$ of the state, with a general increase of the $g$ factor when approaching the circular limit $\beta \rightarrow 1$. However, the states with $N_{1}=N_{2}$ are an exception since, for them, the $g$ factor does not depend on the dot's deformation. This can be understood from the fact that states with $N_{1}=N_{2}$ have no predominant direction of oscillation; as deformation is carried out in a way that the parabola centroid is kept constant $\left[\hbar\left(\omega_{x}\right.\right.$ $\left.\left.+\omega_{y}\right) / 2\right]$ the effects of deformation in one of the principal axes are compensated by the contrary effect on the other principal axis. This compensation does not occur when the state is characterized by an anisotropic oscillation.

\section{CASES OF NUMERICAL TREATMENT}

When the SO coupling cannot be considered weak or when the magnetic field points in a tilted orientation, with respect to the $z$ axis, the above analytical treatment does not remain valid. One must then resort to direct numerical solution of the single-particle Schrödinger equation

$$
h \varphi_{i}(\mathbf{r}, \eta)=\varepsilon_{i} \varphi_{i}(\mathbf{r}, \eta)
$$

As in Ref. 17, we have proceeded by discretizing in a uniform grid of points, finding the orbitals and energies

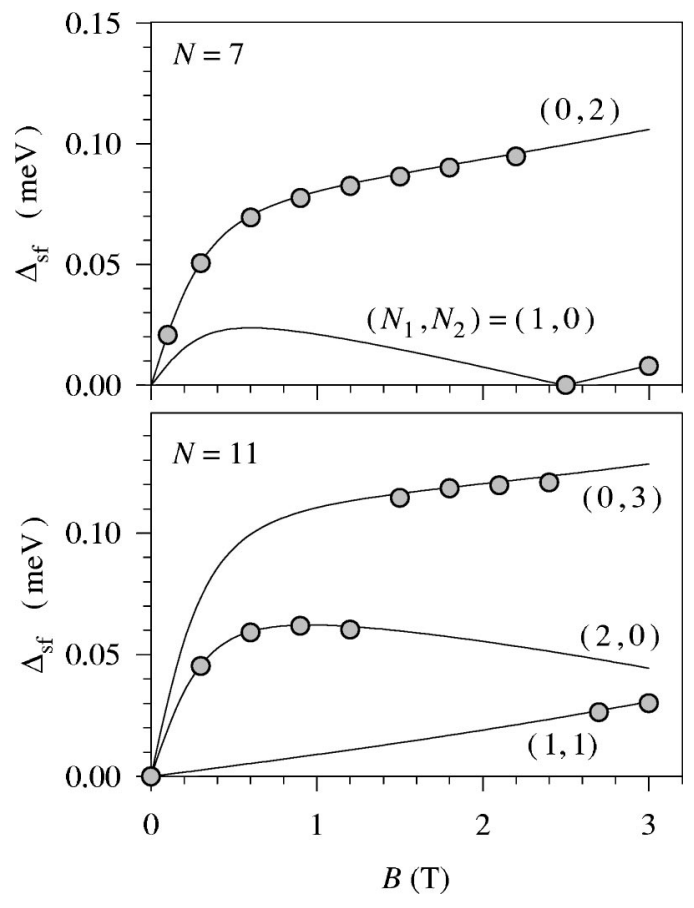

FIG. 4. Numerical results for the spin-flip gap when $\lambda_{R}=0$ and $\lambda_{D}=0.5 \times 10^{-9} \mathrm{eV} \mathrm{cm}$. For comparison the solid lines display the analytical results from Eq. (12). As in the right panel of Fig. 1, the values $\beta=0.9$ and $\hbar\left(\omega_{x}+\omega_{y}\right) / 2=6 \mathrm{meV}$ have been assumed.

$\left\{\varphi_{i}(\mathbf{r}, \eta), \varepsilon_{i}\right\}$ using matrix techniques. In terms of these results one can directly compute the spin-flip strength function,

$$
S_{\text {prec }}(\omega)=\sum_{i j}\left(1-f_{i}\right) f_{j}\left|\left\langle\varphi_{i}\left|\sigma_{x}\right| \varphi_{j}\right\rangle\right|^{2} \delta\left(\varepsilon_{i}-\varepsilon_{j}-\hbar \omega\right)
$$

where $i$ and $j$ span the whole single-particle set and the $f_{i}$ 's give the orbital occupations.

\section{A. Vertical magnetic fields}

We have checked that the numerical solution recovers the previously discussed analytical limit for vertical magnetic fields and weak SO couplings. For instance, Fig. 4 compares the spin-flip gaps for cases with a weak pure Dresselhaus coupling having $N=7$ and 11 electrons. An excellent agreement between the numerical data and the prediction of Eq. (11) is found. Note that in the numerical case discontinuous jumps in the evolution of $\Delta_{s f}$ as a function of $B$ are obtained whenever the ground-state solution implies a reordering of levels in energy. Figure 5 displays a similar result for a pure Rashba coupling, with a somewhat stronger intensity. Small deviations can be seen with the analytical result, although the agreement is still quite good. Our results thus indicate that the analytic treatment works rather well for SO couplings as large as $1.2 \times 10^{-9} \mathrm{eV} \mathrm{cm}$, which is in the range of the experimentally achieved values, and for magnetic fields $B$ $<3 \mathrm{~T}$. 


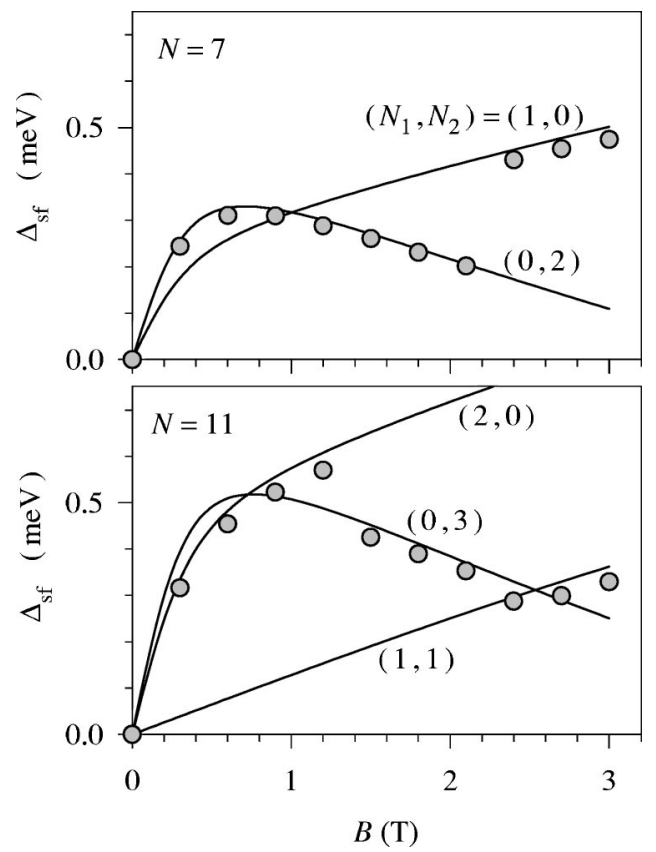

FIG. 5. Same as Fig. 4, but for $\lambda_{R}=1.2 \times 10^{-9} \mathrm{eV} \mathrm{cm}$ and $\lambda_{D}$ $=0$.

\section{B. Tilted magnetic fields}

In Fig. 6 we have analyzed the dependence of the precessional properties on the tilting angle of the magnetic field with respect to the $z$ axis, zero angle meaning perpendicular magnetic field and $\theta=90^{\circ}$ parallel $\mathbf{B}$ to the plane of motion. Note that the spin-orbit interaction is not invariant under rotations in the $x-y$ plane so that its effects depend on the particular direction of tilting. In practice, however, different directions lead to only subtle differences, whilst the strong dependence is given by the angle $\theta$. For this reason we only discuss the case of tilting along the $x$ axis. We find a rather strong dependence of the spin-flip gap on the tilting angle, with a maximum deviation from the Larmor energy for perpendicular field. When the tilting angle is increased a smooth energy decrease in the direction of the Larmor value is seen. Actually, for parallel orientation the results are slightly below the Larmor line. In the lower panel of Fig. 6 the $g$ factors in the limit of vanishing magnetic field are displayed. In correspondence with the transition energies the largest deviations from the bulk value are obtained for the perpendicular direction while the parallel $g$ factor is more similar to the bare factor $(0.44)$. These results can be understood by noting that the SO mechanism couples better with the B-induced currents in the perpendicular geometry ${ }^{19}$ and, therefore, a larger influence on the spin precession is expected in this case.

When the magnetic field has a nonzero vertical component, the spin effects it induces largely dominate over the contribution from the parallel components. For this reason the tilting direction with respect to the dot anisotropy is not very relevant, since the $z$ projection of $\mathbf{B}$ remains the same for different tilting directions. This explains why the results of Fig. 6 are essentially unchanged for tilting directions along the $x$ and $y$ axes of this dot.
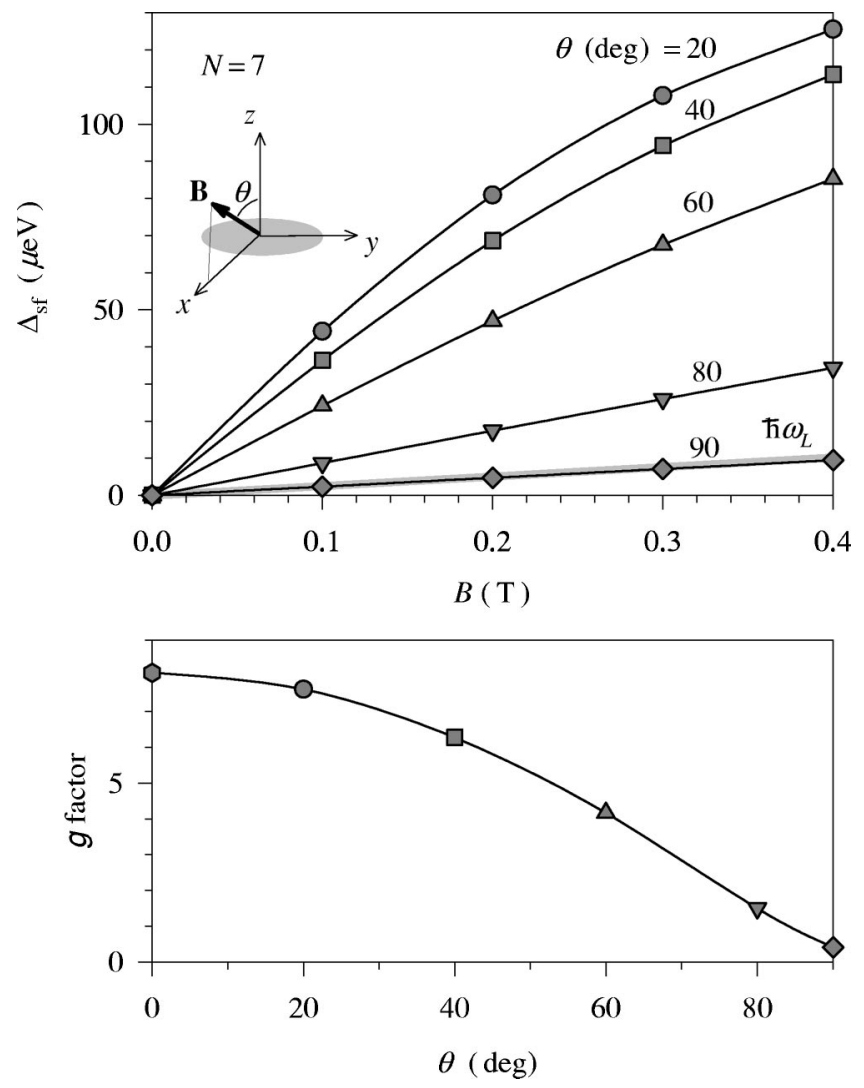

FIG. 6. Upper panel: Dependence of the spin-flip gap on the tilting angle of the magnetic field with respect to the vertical direction. The thick gray line shows the Larmor energy. The values $\beta$ $=0.9$ and $\hbar\left(\omega_{x}+\omega_{y}\right) / 2=6 \mathrm{meV}$ have been assumed. Lower panel: Variation of the $g$ factors in the limit $B \rightarrow 0$ as a function of the tilting angle $\theta$. The assumed SO coupling strengths are $\lambda_{R}=0.35$ $\times 10^{-9} \mathrm{eV} \mathrm{cm}$ and $\lambda_{D}=0.8 \times 10^{-9} \mathrm{eV} \mathrm{cm}$.

\section{A comparison with experiment}

In a recent experiment Hanson et al. ${ }^{8}$ have measured the spin splitting in a one-electron dot by means of conductivity experiments using a parallel magnetic field. It is our purpose here to show that the SO-induced modifications could be the source of the observed deviation of the spin-flip energy with respect to the Larmor result. As stated in the preceding section, when the magnetic field is aligned parallel to the plane of electronic motion the spin splitting recovers a Zeemanlike behavior with an effective $g$ factor slightly smaller than the bulk value. This reduction of the spin splitting is enhanced as the spacing of the orbital levels is reduced, i.e., spin-orbit interaction induces a level repulsion that reduces the spin splittings as the orbital levels get closer.

In Fig. 7 we display the results obtained for a circular one-electron dot (deformation has no significant influence on the spin splitting of the lowest energy state) with feasible values of SO coupling. Namely, we assumed $\lambda_{R}=0.35$ $\times 10^{-9} \mathrm{eV} \mathrm{cm}$, in the range of values mentioned in Refs. 20 and 21 , and $\lambda_{D}=0.8 \times 10^{-9} \mathrm{eV} \mathrm{cm}$. This latter parameter is obtained by assuming a two-dimensional electron gas (2DEG) of width $z_{0} \simeq 60 \AA$ in the formula $\lambda_{D}=\gamma\left(\pi / z_{0}\right)^{2}$, where $\gamma=27.5 \mathrm{eV}^{3}$ is the GaAs specific constant. ${ }^{22}$ The 


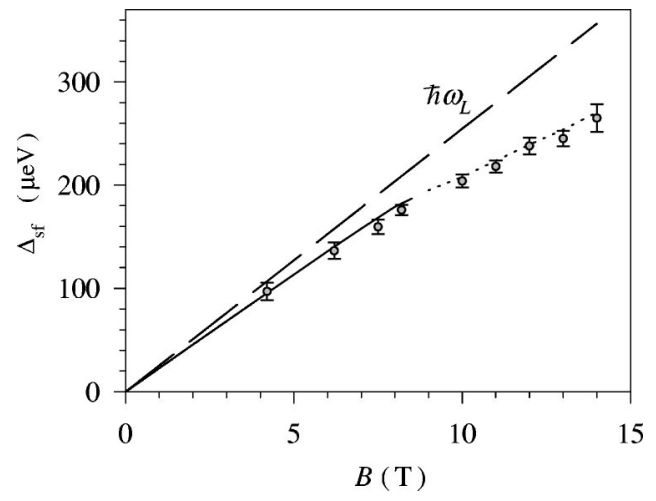

FIG. 7. Experimental spin-flip energy gaps measured in Ref. 8 for a one-electron dot. The solid line is the theoretical result obtained using the experimentally known $\omega_{0}$ values while the dotted extension is a fit (see Sec. III C for a discussion on the additional parameters).

magnetic-field orientation is $45^{\circ}$ above the $x$ axis. We still need to input the external confinement frequency $\hbar \omega_{0}$ before the calculation can be performed. Using for this parameter the measured values of the orbital level spacing, lying between 0.96 and $1.1 \mathrm{meV}$ for the range from $B=0$ to $8 \mathrm{~T}, 23$ one obtains the solid line of Fig. 7. For higher values of the magnetic field (points from $B=8$ to $15 \mathrm{~T}$ ) experimental values of the confinement strength are not available ${ }^{23}$ and we have inferred the $\hbar \omega_{0}$ values in order to fit the measured spin splittings. By assuming $\hbar \omega_{0} \approx 0.5-0.6 \mathrm{meV}$ we obtain the dashed line of Fig. 7. Overall, the agreement with the measurements is rather good and, though this is certainly a simplified model, we believe it indicates that SO coupling plays a role in explaining the measured spin gaps of this system. Obvious extensions of the model would include the explicit consideration of the dimension perpendicular to the plane of the 2DEG and the treatment of nonparabolicity and multiband effects such as, e.g., in the $\mathbf{k} \cdot \mathbf{p}$ model. $^{24}$

We would like to emphasize here that the SO parameter values are sample dependent. The parameter $\lambda_{R}$ is sensitive to an effective electric field in the vertical direction felt by the electrons (affected also by the heterostructure barriers ${ }^{13}$ ) while $\lambda_{D}$ depends on the mean value of the electron vertical momentum squared $\left\langle k_{z}^{2}\right\rangle$ (see, e.g., Ref. 22), estimated above using the effective width $z_{0}$ as $\left\langle k_{z}^{2}\right\rangle \simeq\left(\pi / z_{0}\right)^{2}$. The $\lambda_{R}$ value used in Fig. 7 is reasonably close to the results inferred from the experiments of Refs. 25-27 for GaAs heterostructures. It is necessary to mention, however, that the SO values inferred by Miller et al. ${ }^{15}$ are almost an order of magnitude smaller, a discrepancy that was attributed by these authors to the above-mentioned sample dependence. We end this section by noting that, although the fit in Fig. 7 indeed hints at the larger $\mathrm{SO}$ values, we consider that the number of uncertain ingredients in the present analysis $\left(z_{0}\right.$, electric field, confinement strengths) is too large to draw a definitive conclusion.

\section{Addition of Coulomb interactions}

The above sections have dealt with the SO-induced modifications of the spin precession in the absence of Coulomb

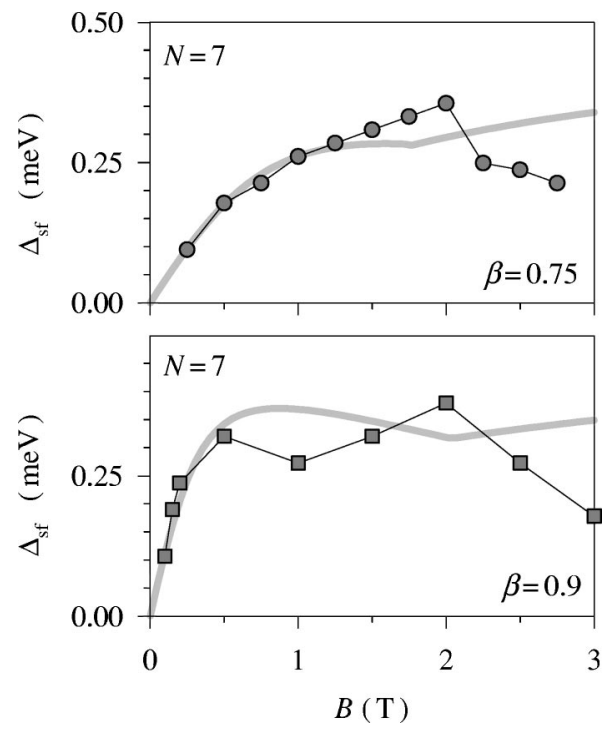

FIG. 8. Spin precessional energies within TDLSDA. The magnetic field points in the vertical $(z)$ direction. As in preceding figures, the centroid value $\hbar\left(\omega_{x}+\omega_{y}\right) / 2$ is fixed at $6 \mathrm{meV}$ while the deformation parameter $\beta$ is given in each panel. The assumed SO coupling strengths are $\lambda_{R}=0$ and $\lambda_{D}=1.2 \times 10^{-9} \mathrm{eV} \mathrm{cm}$. For comparison, the thick gray line shows the analytical result from Sec. II B.

interaction between electrons. We shall now estimate the role of the latter by resorting to the time-dependent local-spindensity approximation (TDLSDA) for noncollinear spins. This approach was already used by us in Ref. 17 for circular dots. The reader is addressed to that reference for more details on this formalism. Here we shall only mention that the integration in time of the TDLSDA equations allows us to monitor the spin precession and, in particular, to extract the precessional frequencies. Since the self-consistent parts of the mean-field potential are recomputed as the system evolves in time one is effectively taking into account dynamical interaction effects. The formalism is thus equivalent to the random-phase approximation (well known in manybody theory) with an effective interaction.

Figure 8 shows for some representative cases the precessional frequencies in TDLSDA with SO coupling and deformation. A vertical magnetic field has been also included. We note that a qualitatively similar behavior is found with respect to the preceding analytical results. In particular, we emphasize that at small $B$ the precessional frequency tends to vanish in the same way as the analytical model for the two $\beta$ 's. Nevertheless, the discontinuity points in the $B$ dependence are different. These sudden jumps are due to level rearrangements induced by the magnetic field. ${ }^{28}$ Since the Coulomb potential modifies the effective mean field it is quite natural that it also affects the transition points. It can be seen that, for the higher deformation (smaller $\beta$ ), the $B$ dependence of the precessional frequencies at low fields is smoother, in agreement with the analytical model. Taking the lowest- $B$ results and using $|g| \equiv \Delta_{s f} /\left(\mu_{B} B\right)$ we obtain $g$ factors of 21.9 and 6.55 for $\beta=0.9$ and 0.75 , respectively, while the corresponding noninteracting values are 20 and 6.2. 


\section{CONCLUSIONS}

In this work we have analyzed the role of the deformation in the confinement to determine, in conjunction with SO coupling and magnetic field, the spin precessional properties of GaAs quantum dots. At small magnetic fields the deformation closes the spin-flip energy gap by allowing the transition between Kramers conjugate states. In practice, this implies that the precessional frequencies of deformed systems have no offsets at $B=0$. The associated $g$ factors depend strongly on the quantum dot electronic state and on the magnetic-field direction. By tilting $\mathbf{B}$ from vertical to horizontal direction one may tune the $g$ factor from large values to results close to the bulk one.

When the magnetic field points in the vertical direction and the SO coupling is weak an analytical treatment, yielding the spin-flip energies and $g$ factors is possible. This provides relevant insights for the analysis of other cases that can only be addressed with numerical approaches. For the case of a one-electron dot in a horizontal magnetic field we have compared our model results with recent experiments. We believe this comparison indicates that the SO coupling plays an important role in explaining the measured spin gaps in this system. For dots containing more electrons, the role of the Coulomb interactions has been estimated within TDLSDA. Sizable modifications of the single-particle picture have been obtained but the agreement is good in the limit of vanishing magnetic fields. The main features of the analytical model are qualitatively preserved within TDLSDA.

\section{ACKNOWLEDGMENTS}

This work was supported by Grant No. BFM2002-03241 from DGI (Spain). We thank R. Hanson for useful discussions and for providing us the data used in Fig. 7.
${ }^{1}$ S.A. Wolf, D.D. Awschalom, R.A. Buhrman, J.M. Daughton, S. von Molnár, M.L. Roukes, A.Y. Chtchelkanova, and D.M. Treger, Science 294, 1488 (2001).

${ }^{2}$ M. Ciorga, A.S. Sachrajda, P. Hawrylak, C. Gould, P. Zawadzki, S. Jullian, Y. Feng, and Z. Wasilewski, Phys. Rev. B 61, R16315 (2000).

${ }^{3}$ S. Datta and B. Das, Appl. Phys. Lett. 56, 665 (1990).

${ }^{4}$ M. Valín-Rodríguez, A. Puente, Ll. Serra, and E. Lipparini, Phys. Rev. B 66, 235322 (2002).

${ }^{5}$ D.D. Awschalom and J.M. Kikkawa, Phys. Today 52 (6), 33 (1999).

${ }^{6}$ J.A. Gupta, D.D. Awschalom, X. Peng, and A.P. Alivisatos, Phys. Rev. B 59, 10421 (1999); J.A. Gupta, D.D. Awschalom, Al.L. Efros, and A.V. Rodina, ibid. 66, 125307 (2002).

${ }^{7}$ T. Fujisawa, D.G. Austing, Y. Tokura, Y. Hirayama, and S. Tarucha, Nature (London) 419, 278 (2002).

${ }^{8}$ R. Hanson, B. Witkamp, L.M.K. Vandersypen, L.H. Willems van Beveren, J.M. Elzerman, and L.P. Kouwenhoven, Phys. Rev. Lett. 91, 196802 (2003).

${ }^{9}$ A.V. Khaetskii and Y.V. Nazarov, Phys. Rev. B 64, 125316 (2001)

${ }^{10}$ O. Voskoboynikov, C.P. Lee, and O. Tretyak, Phys. Rev. B 63, 165306 (2001).

${ }^{11}$ T. Koga, J. Nitta, H. Takayanagi, and S. Datta, Phys. Rev. Lett. 88, 126601 (2002).

${ }^{12}$ T. Koga, J. Nitta, T. Akazaki, and H. Takayanagi, Phys. Rev. Lett. 89, 046801 (2002).

${ }^{13}$ D. Grundler, Phys. Rev. Lett. 84, 6074 (2000).

${ }^{14}$ D.M. Zumbühl, J.B. Miller, C.M. Marcus, K. Campman, and A.C. Gossard, Phys. Rev. Lett. 89, 276803 (2002).

${ }^{15}$ J.B. Miller, D.M. Zumbühl, C.M. Marcus, Y.B. Lyanda-Geller, D.
Goldhaber-Gordon, K. Campman, and A.C. Gossard, Phys. Rev. Lett. 90, 076807 (2003).

${ }^{16}$ I.L. Aleiner and V.I. Fal'ko, Phys. Rev. Lett. 87, 256801 (2001).

${ }^{17}$ M. Valín-Rodríguez, A. Puente, Ll. Serra, and E. Lipparini, Phys. Rev. B 66, 165302 (2002).

${ }^{18}$ We characterize the spin-flip absorption strength for a transition between orbital $\left|N_{1} N_{2} \eta\right\rangle$ and $\left|N_{1}^{\prime} N_{2}^{\prime} \eta^{\prime}\right\rangle$ by $\left|\left\langle N_{1} N_{2} \eta\left|\sigma_{x}\right| N_{1}^{\prime} N_{2}^{\prime} \eta^{\prime}\right\rangle\right|^{2}$.

${ }^{19}$ Note that only the vertical component of the magnetic field explicitly appears in the spin-orbit terms $h_{R}$ and $h_{D}$, Eqs. (5) and (6), through the horizontal kinetic momenta $P_{x}$ and $P_{y}$.

${ }^{20}$ I.D. Vagner, A.S. Rozhavsky, P. Wyder, and A.Yu. Zyuzin, Phys. Rev. Lett. 80, 2417 (1998).

${ }^{21}$ Yu.A. Bychkov and E.I. Rashba, J. Phys. C 17, 6039 (1984).

${ }^{22}$ W. Knap, C. Skierbiszewski, A. Zduniak, E. Litwin-Staszewska, D. Bertho, F. Kobbi, J.L. Robert, G.E. Pikus, F.G. Pikus, S.V. Iordanskii, V. Mosser, K. Zekentes, and Yu.B. Lyanda-Geller, Phys. Rev. B 53, 3912 (1996).

${ }^{23}$ R. Hanson (private communication).

${ }^{24} \mathrm{~T}$. Wenckebach, Essentials of Semiconductor Physics (Wiley, Chichester, UK, 1999).

${ }^{25}$ D. Stein, K.v. Klitzing, and G. Weimann, Phys. Rev. Lett. 51, 130 (1983).

${ }^{26}$ B. Jusserand, D. Richards, H. Peric, and B. Etienne, Phys. Rev. Lett. 69, 848 (1992).

${ }^{27}$ P. Ramvall, B. Kowalski, and P. Omling, Phys. Rev. B 55, 7160 (1997).

${ }^{28}$ We have checked that the numerical results are converged with respect to the spatial step of the grid. Additionally, when SO coupling is removed we obtain the Larmor frequency $\omega_{L}$ with an accuracy better than $1 \%$. 